\title{
The calcineurin/NFAT pathway is activated in diagnostic breast cancer cases and is essential to survival and metastasis of mammary cancer cells
}

\author{
C Tran Quang ${ }^{*, 1,2,3}$, S Leboucher ${ }^{1,2,3}$, D Passaro ${ }^{1,2,3}$, L Fuhrmann ${ }^{4,5,6}$, M Nourieh $^{4,6}$, A Vincent-Salomon ${ }^{4,6,7}$ and J Ghysdael ${ }^{*, 1,2,3}$
}

Nuclear factor of activated T cells 1 (NFAT1) expression has been associated with increased migratory/invasive properties of mammary tumor-derived cell lines in vitro. It is unknown, however, if NFAT activation actually occurs in breast cancer cases and whether the calcineurin/NFAT pathway is important to mammary tumorigenesis. Using a cohort of 321 diagnostic cases of the major subgroup of breast cancer, we found Cn/NFAT pathway activated in ER ${ }^{-} \mathrm{PR}^{-} \mathrm{HER} 2^{-}$triple-negative breast cancer subtype, whereas its prevalence is less in other subgroups. Using a small hairpin RNA-based gene expression silencing approach in murine mammary tumor cell line (4T1), we show that not only NFAT1 but also NFAT2 and their upstream activator $\mathrm{Cn}$ are essential to the migratory and invasive properties of mammary tumor cells. We also demonstrate that Cn, NFAT1 and NFAT2 are essential to the tumorigenic and metastatic properties of these cells in mice, a phenotype which coincides with increased apoptosis in vivo. Finally, global gene expression analyses identified several NFAT-deregulated genes, many of them being previously associated with mammary tumorigenesis. In particular, we identified the gene encoding a disintegrin and metalloproteinase with thrombonspondin motifs 1 , as being a potential direct target of NFAT1. Thus, our results show that the Cn/NFAT pathway is activated in diagnostic cases of breast cancers and is essential to the tumorigenic and metastatic potential of mammary tumor cell line. These results suggest that pharmacological inhibition of the Cn/NFAT pathway at different levels could be of therapeutical interest for breast cancer patients.

Cell Death and Disease (2015) 6, e1658; doi:10.1038/cddis.2015.14; published online 26 February 2015

Breast cancer is the first cause of death by cancer of women in developed countries. Its progression is characterized by distinct steps, starting with hyperplasia, in situ ductal carcinoma and invasive carcinoma, which can evolve into a deadly metastatic disease. Breast cancer is a complex disease in which tumor maintenance and progression to invasiveness relies both on tumor cell-intrinsic genetic lesions in oncogenes and tumor suppressor genes and on a constant dialog between tumor cells and their microenvironment. ${ }^{1}$

The nuclear factor of activated T cells (NFAT) family of transcription factors is composed of four $\mathrm{Ca} 2+$-regulated members (NFAT1-4) and one member regulated in response to osmotic stress (NFAT5). First identified as regulators of IL2 gene transcription in activated T cell, NFAT factors have since been shown essential to the development of diverse tissues (for review, see references Macian ${ }^{2}$ and Muller and $\mathrm{Rao}^{3}$ ). Classically, in unstimulated T cells, NFAT1-4 proteins reside in the cytoplasm in an hyperphosphorylated form. Activation of cell surface receptors coupled to $\mathrm{Ca} 2+$ mobilization from intracellular stores and ensuing opening of calcium-release activated channels (CRAC) leads to the activation of $\mathrm{Ca} 2$ +-dependent enzymes, in particular, the calcineurin $(\mathrm{Cn})$ protein phosphatase. Once activated, Cn catalyzes NFAT dephosphorylation, leading to its nuclear translocation. In the nucleus, NFAT factors regulate gene transcription, often in cooperation with unrelated transcriptional regulators. Cessation of $\mathrm{Cn}$ activation leads to the sequential rephosphorylation of nuclear NFAT by specific kinases and its export to the cytoplasm.

The implication of NFAT in oncogenic processes is beginning to emerge. First, the expression of a constitutively nuclear mutant of NFAT2 in immortalized 3T3 L1 fibroblasts leads to their transformation, suggesting an intrinsic role for NFAT in cellular transformation. ${ }^{4}$ Second, deregulation of NFAT expression or nuclear accumulation has been observed in several pathologies such as pancreatic, ${ }^{5,6}$ prostate $^{7}$ and in lymphoid malignancies. ${ }^{8,9}$ In T-cell acute lymphoblastic leukemia (T-ALL), $\mathrm{Cn}$ is critical to the propagating activity of leukemic cells and controls nuclear accumulation of NFAT. ${ }^{9,10}$ In breast carcinoma-derived cell lines, an Akt-dependent pathway regulating NFAT1 proteolytic degradation and in vitro cell migration and invasion has been described. ${ }^{11}$ Yet, the involvement of $\mathrm{Cn}$ in NFAT1 activation in this context is not established. ${ }^{12}$ Most importantly, the relevance of the activation of the Cn/NFAT module to breast cancer biology in vivo

\footnotetext{
${ }^{1}$ U1005-UMR3306-, Institut Curie, Bat 110 Centre Universitaire, Orsay 91405, France; ${ }^{2}$ Institut National de la Recherche Santé et de la Recherche Medicale, Orsay U1005, France; ${ }^{3}$ Centre National de la Recherche Scientifique, Orsay UMR3306, France; ${ }^{4}$ Centre de Recherche, Institut Curie, Paris 75005, France; ${ }^{5} \mathrm{CNRS}$ UMR144, Paris 75005, France; ${ }^{6}$ Department of Biopathology, Institut Curie, Paris 75005, France and ${ }^{7}$ INSERM U934, Paris 75005, France

*Corresponding authors: C Tran Quang or J Ghysdael, U1005-UMR3306-, Institut Curie, Bat 110 Centre Universitaire, Orsay 91405, France. Tel: +33 169863023 ; Fax: +3316986 31 52; Email: Christine.Tran-Quang @ curie.fr or Jacques.Ghysdael@ curie.fr

Abbreviations: NFAT, nuclear factor of activated T cells; CnB1, calcineurin B1; T-ALL, T-cell acute lymphoblastic leukemia; ADAMTS1, A Disintegrin And Metalloproteinase with ThromboSpondin motifs 1; TNBC, triple-negative breast cancer; ER, estrogen receptor; PR, progesterone receptor; HER2, human epidermal growth factor receptor-2

Received 17.9.14; revised 23.12.14; accepted 07.1.15; Edited by G Dewson
} 
remains to be determined. To address these questions, we investigated whether the Cn/NFAT pathway is activated in diagnostic cases of breast cancer, and found Cn/NFAT module to be frequently activated in $\mathrm{ER}^{-} \mathrm{PR}^{-} \mathrm{HER} 2^{-}$triple-negative molecular poor prognostic subgroup. Using the 4T1 triplenegative mammary cell line, we show that NFAT1 or NFAT2 silencing impair the migration and invasion properties of tumor cells and that both NFAT1 and NFAT2 act downstream of Cn. Transcriptomic analysis identified over 300 genes, which are significantly deregulated in silenced NFAT1 cells, many of them being implicated in mammary tumorigenesis. In particular, we report that expression of the protease A Disintegrin And Metalloproteinase with ThromboSpondin motifs 1 (ADAMTS1), which was previously shown to be essential to mammary tumor development and metastasis, ${ }^{13,14}$ is likely a direct target of NFAT1.

\section{Results}

The Cn/NFAT pathway is frequently activated in the triplenegative breast cancer subgroup. To investigate the activation status of Cn/NFAT module in breast cancer, we analyzed the expression and subcellular localization of NFAT in 321 primary breast tumors representative of the four main molecular subtypes of breast cancer (See Supplementary Table 1 for patients clinicopathological characteristics). As shown in Figures 1a and b, nuclear NFAT2 was detected in 42/83 of the $\mathrm{ER}^{-} \mathrm{PR}^{-} \mathrm{HER}^{-}$(TNBC; triple-negative breast cancer) tumors, whereas only a minority of the luminal $A$, luminal B and HER2+ tumors showed nuclear NFAT2 staining (12/101, 16/85 and 4/52, respectively). NFAT1 was also found nuclear in about half of the NFAT2-positive TNBC biopsies (see Supplementary Figure 1 for an example of NFAT1 nuclear staining). The $\mathrm{H}$ score of nuclear NFAT2, which takes into consideration the staining intensity in conjunction with the percentage of positively stained cells, was also found increased in $\mathrm{ER}^{-} \mathrm{PR}^{-} \mathrm{HER} 2^{-}$tumors as compared with the three other molecular subtypes (Figure 1c). These data show that nuclear accumulation of NFAT is observed in the most aggressive subtype of breast cancer. In line with this, the $\mathrm{H}$ score of nuclear NFAT2 was found higher in grade 3 tumors, which show the highest proliferative index and a highest disorganized architecture (Figure 1d). These results highlight the activation status of the Cn/NFAT pathway in the worst prognostic cases of breast cancer.

CnB1, NFAT1 or NFAT2 silencing inhibits cell migration/ invasion in vitro. Previous studies reported that NFAT1 is endowed with pro-migratory and pro-invasive capacities in breast cancer cell lines in vitro (see Introduction). We thus investigated whether this property is specific to NFAT1 and whether it depends on $\mathrm{Cn}$ activation. For this, the 4T1 mammary tumor cell line was stably transduced with lentiviruses expressing NFAT1-, NFAT2-, CnB1-specific small hairpin RNA (shRNA) or the pLKO as control. As shown in Figure $2 \mathrm{a}$, this resulted in efficient and stable knockdown of the expression of the respective proteins. Of note, $\mathrm{CnB} 1$ knockdown resulted in NFAT1 and NFAT2 rephosphorylation, also observed in cells treated with a $\mathrm{Cn}$ inhibitor (Figure 2b).
This indicates that Cn controls NFAT activation in mammary tumor cells. Inhibition of the Cn/NFAT signaling module in cells grown in vitro in $10 \%$ FBS did not affect their maintenance as no significant difference was observed between the expansion of control cells or cells silenced for either CnB1, NFAT1 or NFAT2 (Figure 2c). In contrast, under these conditions, CnB1, NFAT1 or NFAT2 silencing inhibited motility of 4T1 cells as assessed in Boyden chamber assays and by time lapse video microscopy (Figure 2d; Supplementary Figure 2 and 3) and impaired their ability to heal a wound (Supplementary Figure 3). Of note, the data of Supplementary Figure 3 used a distinct set of shRNAs for CnB1, NFAT1 or NFAT2, thus excluding nonspecific, off-target effects of the shRNAs used. Invasion was also analyzed using Boyden chambers with membranes precoated with a layer of Matrigel. CnB1, NFAT1 or NFAT2 knockdown also resulted in impaired invasion (Figure $2 \mathrm{e}$ ). These results show that $\mathrm{Cn}$ and its major effectors NFAT1 and NFAT2 are important for breast cancer cells migration and invasion.

The Cn/NFAT pathway is essential for mammary tumorigenesis. To next determine whether the Cn/NFAT pathway is essential to tumorigenesis, the control and CnB, NFAT1 or NFAT2-silenced 4T1 cells were injected into the mammary gland of syngenic Balb/c mice and tumor growth was monitored every 3 days over a period of 3-6 weeks, time after which mice were killed. Immunohistochemical analysis of NFAT1 in 4T1 tumors revealed the activation of the $\mathrm{Cn}$ / NFAT pathway, as NFAT1 was detected in the nuclei (black stars) of control tumor cells, whereas it was exclusively found in the cytoplasm of shCnB1-silenced tumors and not detected in shNFAT1-silenced tumors (Figure 3a). As shown in Figures $2 \mathrm{~b}$ and $\mathrm{c}$, CnB1, NFAT1 or NFAT2 silencing resulted in a severe decrease in tumor size. Similar experiments realized with 168FARN cells, a less metastatic murine mammary tumor cell line, also show that NFAT1 or NFAT2 silencing resulted in decreased tumor size (data not shown). To understand the basis of the decreased size of CnB1-, NFAT1- and NFAT2-silenced tumors, tumors were analyzed 5 or 15 days after orthotopic engraftment for proliferation and apoptosis by Ki67 and cleaved caspase 3 immunohistochemical staining, respectively. No significant difference was observed in the proliferation/apoptosis rates, soon after cells injection (day 5, data not shown). Similarly, 2 weeks after engraftment, no difference was found in the proliferation rate of the different tumors (Figure $3 d$, upper panel). In contrast, a twofold increase in apoptotic cells was seen in CnB1-, NFAT1- and NFAT2-silenced tumors as compared with tumors transduced with the control vector (Figures $3 d$ and e). These observations indicate that $\mathrm{Cn}$, NFAT1 and NFAT2 are essential components of a survival pathway activated in mammary tumors in vivo.

The Cn/NFAT pathway is essential for metastasis. In line with previous studies, ${ }^{15}$ metastatic cells were clearly detected in the lymph nodes, lungs and bones of mice carrying control tumors with an average size of $600 \mathrm{~mm}^{3}$ (see supplementary Table 2). In contrast, metastasis was profoundly inhibited in mice carrying similar sized CnB1, NFAT1 or NFAT2-silenced tumors, suggesting a function of $\mathrm{Cn}$ and NFAT in metastasis 
a

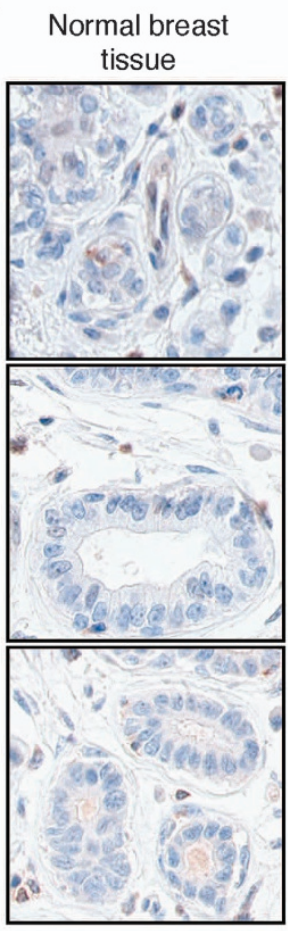

Tumor ER-PR-HER2-

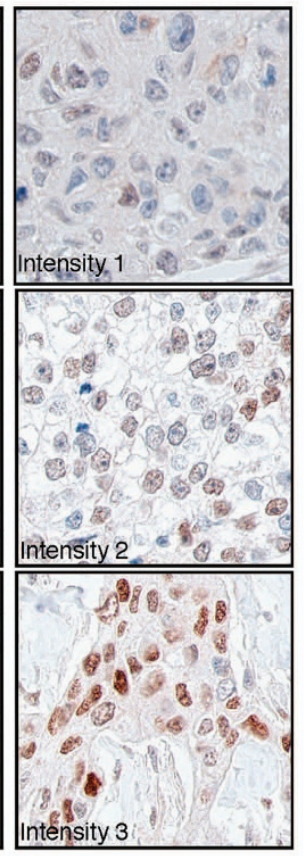

b

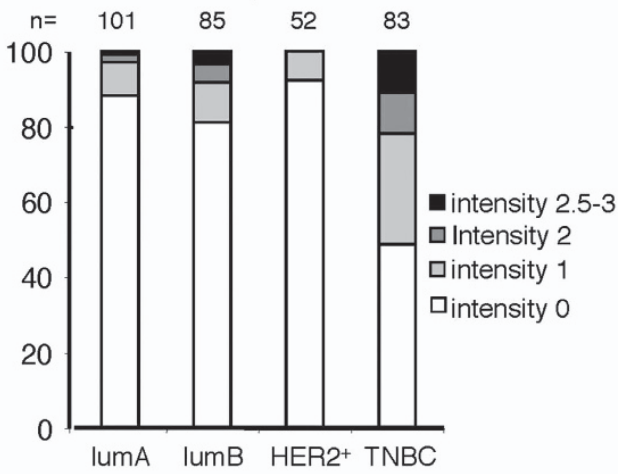

C

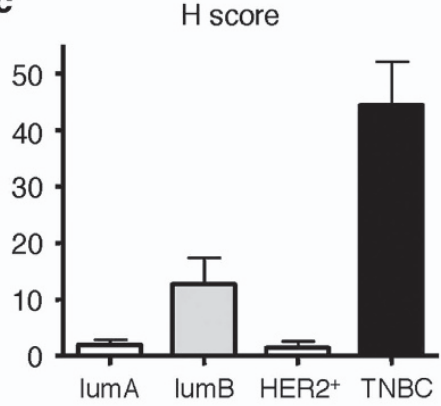

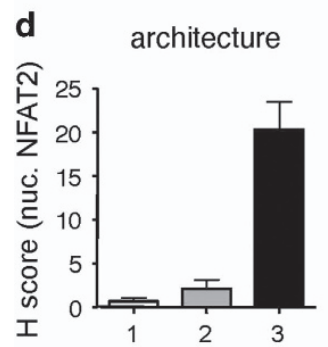

anisocaryosis

mitotic index

grade
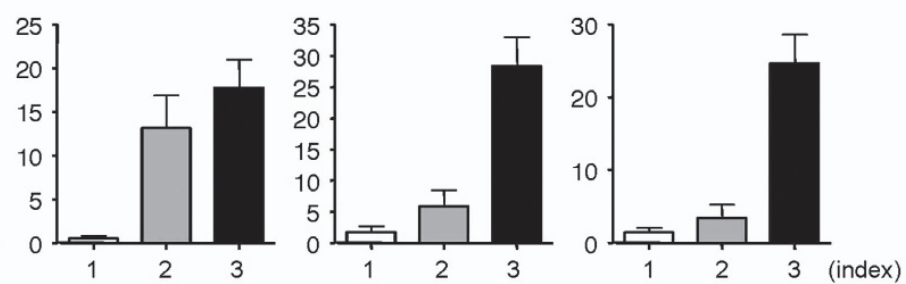

Figure 1 Nuclear localization of NFAT2 in hormone receptor-negative tumors. (a) Representative immunohistochemical staining of NFAT2 in sections of human breast tumors TMAs showing adjacent non neoplastic tissues (left panels) and ER ${ }^{-} \mathrm{PR}^{-} \mathrm{HER} 2^{-}$triple-negative tumors showing different intensity staining. (b) Intensity scoring of nuclear NFAT2 immunohistochemistry staining of tissue microarray (TMAs) of human breast tumors representative of the different molecular subtypes defined for their positivity to specific markers as follows: lumA: estrogen receptor (ER) $>10 \%$, progesterone receptor $(\mathrm{PR})>20 \%$, Ki67 $<14 \%$; LumB: ER $>10 \%, \mathrm{PR}<20 \%, \mathrm{Ki} 67>14 \%$; Her2+: ER $<10 \%$, $\mathrm{PR}<10 \%$, HER2 2+ amplified; TNBC (triple negative): $\mathrm{ER}<10 \%, \mathrm{PR}<10 \%, \mathrm{HER} 20 / 1$ or $2+$ non amplified according to ASCO guideline. The number of cases analyzed in the different subtypes is indicated at the top. (c) Nuclear NFAT2 H score (intensity $\times$ percentage of positively stained cells) in the different molecular subtype of breast cancer. (d) Nuclear NFAT2 $\mathrm{H}$ score according to the architecture, anisocaryosis, proliferative index and grade of the tumors

(Supplementary Table 2). To address more directly whether the $\mathrm{Cn}$ pathway is essential to the metastatic potential of breast cancer cells, 4T1 cells and its derivatives were directly injected in the tail vein of Balb/c mice. After 2 weeks, mice were killed and metastatic burden was analyzed. As shown in Figure $4 \mathrm{a}$, mice injected with control 4T1 cells presented an increased weight of the lungs as compared with control mice $(0.40 \pm 0.02 \mathrm{~g}$ versus $0.14 \pm 0.01 \mathrm{~g})$, which correlated with the massive focal colonization of lungs by tumor cells (Figures $4 b$ and c). In contrast, CnB1-, NFAT1-, NFAT2-silenced 4T1 cells were severely impaired in their ability to expand in lungs (Figure 4a), also shown in histological analysis (Figures 4b and c). Similarly to what was observed in primary tumors, an increase in apoptotic cells was seen in the metastatic nodules generated from NFAT1- and NFAT2-silenced 4T1 cells as compared with controls (Figures $4 \mathrm{~b}$ and $\mathrm{d}$ ). These results show that $\mathrm{Cn}$ and its NFAT1 and NFAT2 effectors are important both during the tumorigenic and metastatic processes, in part through their antiapoptotic properties.

\section{Characterization of the NFAT1-dependent transcriptome} in 4T1 cells. To investigate the molecular basis of NFAT prooncogenic function, the transcriptome of control and NFAT1silenced cells were compared using pangenomic mouse GeneChip 4302.0 arrays (Affymetrix). Unsupervised clustering analyses showed that three independently generated control 4T1 cell cultures clustered together and away from the cluster formed by three independent shNFAT1-4T1 cultures (Figure 5a). To identify NFAT-dependent biological pathways and functions, we conducted gene ontology and pathway analyses using the 325 genes that showed an at least twofold expression change, using ingenuity pathways analysis (IPA). Major functions expected to be essential to the tumorigenic potential of cells were significantly affected, including 'cellular 
a

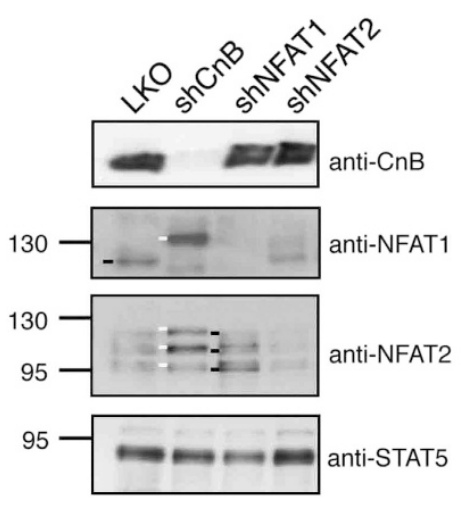

d

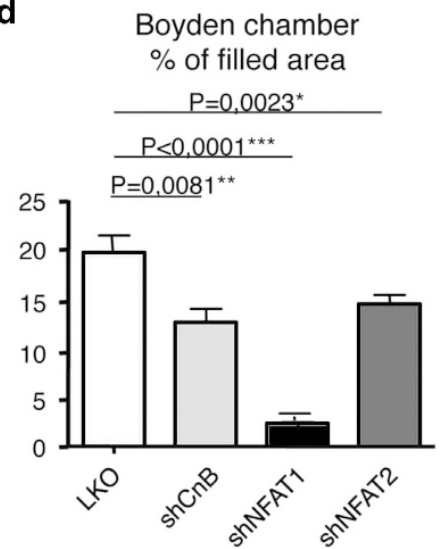

b

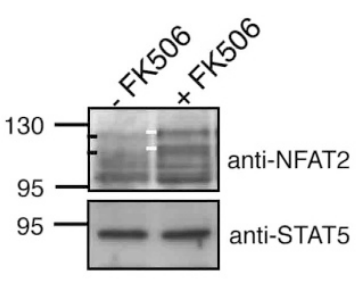

c

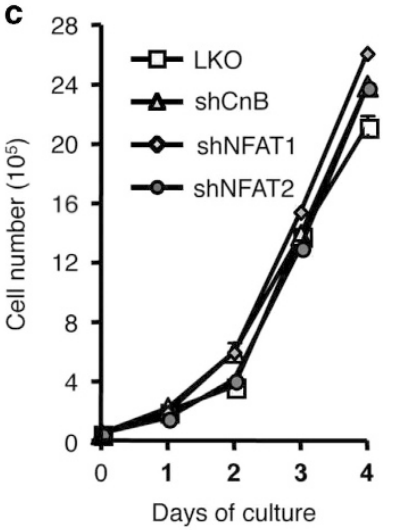

e

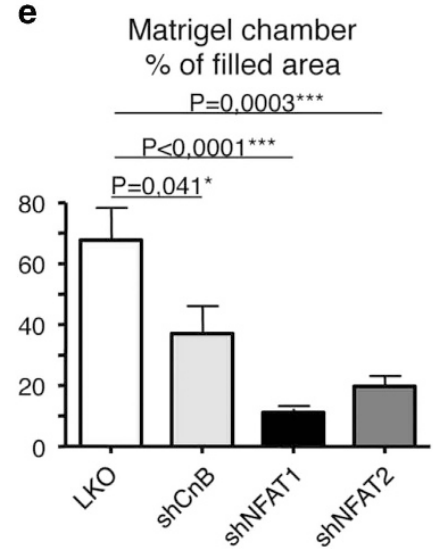

Figure 2 Silencing of CnB1, NFAT1 or NFAT2 in 4T1 cell impairs cells migration and invasion. (a) Extracts of shRNA-transduced and puromycin-resistant 4T1 cells, maintained for 1 week in culture, were immunoblotted for $\mathrm{CnB1}$ (upper panel), NFAT1 (upper intermediate panel) or NFAT2 (lower intermediate panel) expression. The white traits point to the hyperphosphorylated isoforms of NFTA1 and 2, whereas black traits point to active, dephosphorylated isoforms of NFAT1 and 2. The blots were probed for STAT5 as normalizer. A representative blot of at least three independent experiment is shown. (b) As in (a) except using extract of $4 \mathrm{~T} 1 \mathrm{cells}$ treated or not for $24 \mathrm{~h}$ with $50 \mathrm{nM}$ FK506. (c) Growth of control and the indicated 4T1 cell derivatives maintained in culture in DMEM/F12+10\% of FBS ( $n=3$, data are represented as mean \pm S.E.M.). (d) $5 \times 10^{4}$ of the indicated cells cultured in the absence of serum were seeded in the upper compartment of a Boyden chamber and assessed for their ability to migrate toward the lower compartment containing $10 \%$ FBS. After $16 \mathrm{~h}$, the membranes were stained for the migratory cells and quantification of surface areas covered by migrating cells, performed using ImageJ. Data are the means of three independent experiments $\left(n=3\right.$, data are represented as mean \pm S.E.M.). (e) As in (d) except $2 \times 10^{5}$ cells were seeded for $24 \mathrm{~h}$ in modified Boyden Chamber loaded with Matrigel

growth and proliferation', 'cell death and survival' and 'cell movement' (Table 1). As apoptosis was enhanced in vivo in NFAT1-silenced tumors, we analyzed in more details the set of genes ascribed to this phenotype. Table 2 shows the top 10 downregulated $(-)$ and upregulated $(+)$ genes in shNFAT1$4 \mathrm{~T} 1$ cells as compared with control cells. Interestingly, $70 \%$ of these deregulated genes (14 out of 20) were previously reported as important to mammary tumorigenesis (indicated by the asterisks in Table 2), reinforcing the hypothesis of an essential role of the Cn/NFAT pathway in breast cancer. The deregulated expression of ADAMTS1, ROR1, FST, TXNIP and KLF2 between control and NFAT1-silenced 4T1 cells was confirmed by semi-quantitative RT-PCR (supplementary Figure 4). Interestingly, the same trend was observed for these genes in CnB1 and NFAT2-silenced cells, suggesting common molecular targets between Cn, NFAT1 and NFAT2 (supplementary Figure 4). We further focused on ADAMTS1, as this gene, which encodes a protease involved in mammary tumor growth and metastasis, ${ }^{13,14}$ was found among the most downregulated genes in NFAT-silenced cells and also ascribed to cell movement in IPA analysis (data not shown). RT-PCR analysis independently confirmed the strong downregulation of ADAMTS1 expression in NFAT1 4T1 cells (Figure $5 \mathrm{~b}$ ). The promoter region of mouse ADAMTS $1^{16}$ revealed the presence of a NFAT consensus DNA-binding site $A / T$ GGAAA (A/N) (A/T/C) N. Chromatin immunoprecipitation using an anti-NFAT1 antibody was thus performed to investigate NFAT1 binding to ADAMTS1 promoter region. For this, control and shNFAT1-4T1 cells were either maintained under steady-state conditions or stimulated with PMA/ lonomycin to ensure full NFAT1 activation. As shown in Figure $5 \mathrm{c}$, the promoter region of ADAMTS1 was recovered specifically from the NFAT1 immunoprecipitates of $4 T 1$ cells, but not from the chromatin immunoprecipitates of NFAT1silenced 4T1 cells. These results suggest that ADAMTS1 expression is under direct NFAT1 transcriptional control. As the pro-metastatic properties of ADAMTS1 have been shown to rely on its metallopeptidase activity ${ }^{17}$ and as ADAMTS1 is endowed with gelatinase activity, ${ }^{18}$ we next compared the ability of $4 \mathrm{~T} 1$ and NFAT1-silenced $4 \mathrm{~T} 1$ cells to degrade 
glutaraldehyde cross-linked gelatin. For this, cells were plated on FITC-gelatin and disappearance of fluorescence analyzed. As shown in Figure $5 d$ and quantified in Figure 5e, control cells displayed large spots of digested gelatin, whereas NFAT1-silenced cells showed a decreased number of digested areas. This suggests that the inability of

a

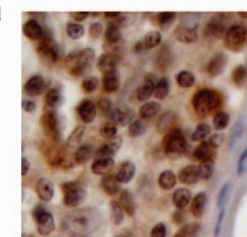

Lymph node

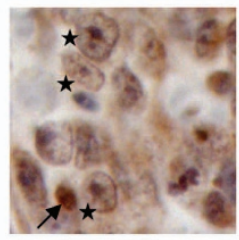

LKO

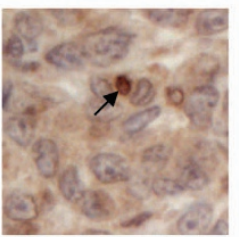

$\operatorname{shCnB}$

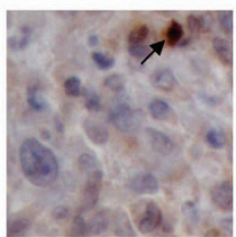

shNFAT1 b

d
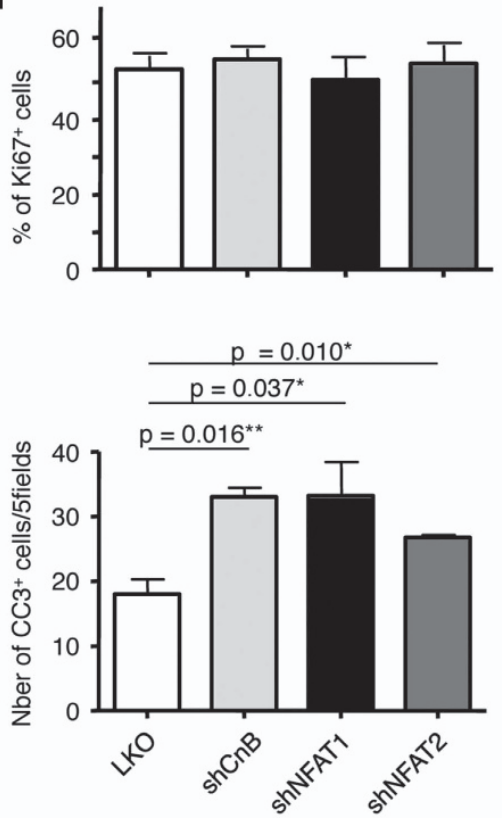

C

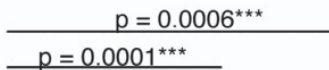

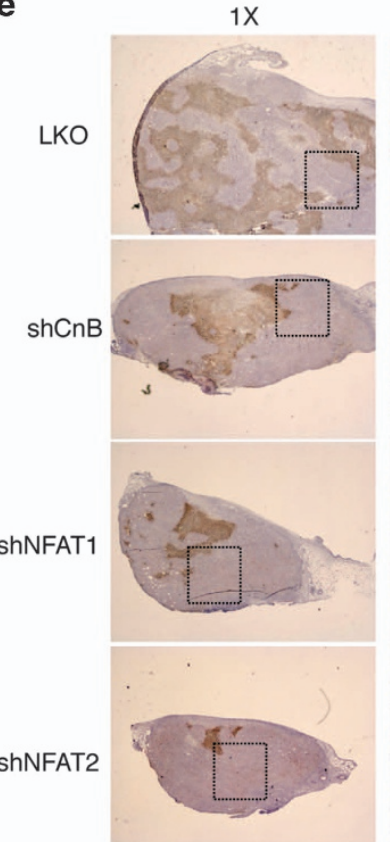

$20 X$

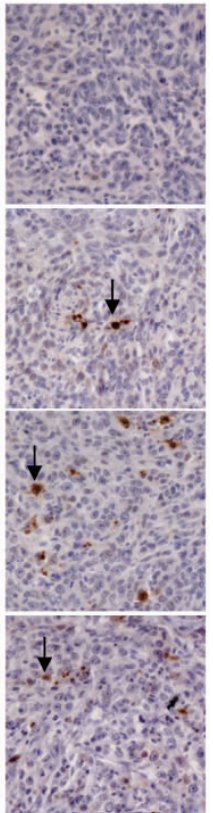

Figure 3 Knockdown expression of CnB, NFAT1 or NFAT2 impairs in vivo tumor growth. (a) Immunohistochemical analysis of NFAT1 expression and subcellular localization in control (LKO), CnB1-silenced (shCnB) and NFAT1-silenced (shNFAT1) 4T1 tumors. A lymph node section of control mice (left panel) has been used as positive control for NFAT1 expression and nuclear localization. Arrows point to NFAT1-expressing lymphoid infiltrating cells, whereas stars point to nuclear NFAT1 in mammary tumor cells. (b) $4 \mathrm{~T} 1$ cells $\left(5 \times 10^{5} ; n=4\right.$ for each $4 \mathrm{~T} 1$ cell derivatives) were inoculated in the inguinal mammary fat pad of syngeneic Balb/c mice. Tumor size was measured every 3 days with a caliper and the tumor volume calculated according to the equation $V=\left(L \times W^{2}\right) / 2$. Data are represented as mean \pm S.E.M. (c) Tumor volume at the time of killing, 3-5 weeks after inoculation. Control pLKO tumors (white dots, $n=21$ ), shCnB1 tumors (light gray, $n=21$ ), shNFAT1 tumors (black dots, $n=21$ ), shNFAT2 tumors (gray dots, $n=16$ ). Data are represented as mean \pm S.E.M. (d) Quantification of the number of proliferative cells (Ki67+, upper panel) and apoptotic cells (CC3+, lower panel) in tumors ( $n=3$ ) 15 days after inoculation. Numbers are the results of counting five consecutive fields at the $\times 20$ magnification. Data are represented as mean \pm S.E.M. (e) Tumors excised 15 days after orthotopic injection were fixed, paraffin embedded and further analyzed for the expression of cleaved caspase 3 (CC3) by immunohistochemistry. The right panels represent a higher magnification of the insets shown in respective left panels. A representative picture of each tumor is shown. Arrows point to CC3-positive cells. Note the unspecific brown staining of necrotic areas observed at the $\times 1$ magnification 
a

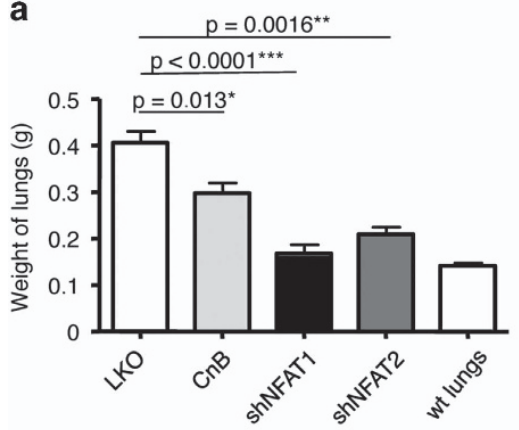

b

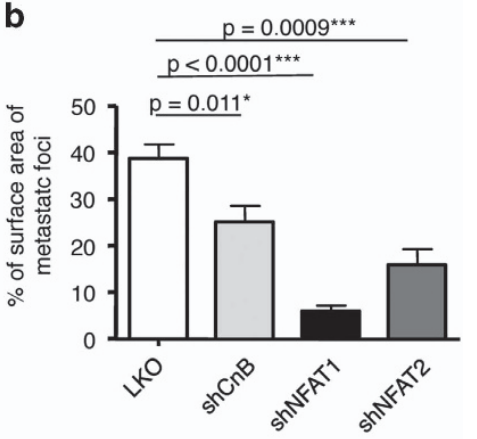

C

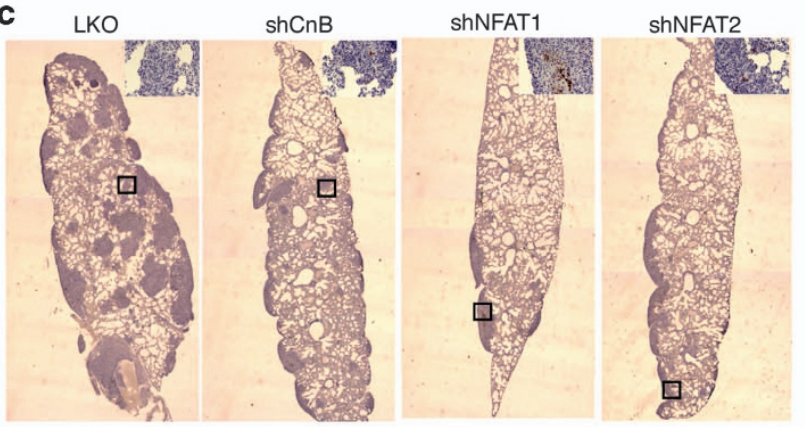

d

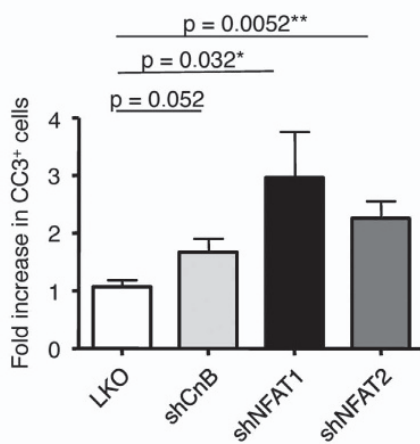

Figure 4 Inactivation of either Cn, NFAT1 or NFAT2 impairs the metastatic potential of 4T1 cells. (a) Weight of the lungs excised from killed mice, 2 weeks after intravenous injection of $5 \times 10^{5} 4 \mathrm{~T} 1$ cells. Note the significant decrease of the lung weight of mice injected with shCnB1-transduced cells $(0.29 \pm 0.02 \mathrm{~g}, n=5)$, shNFAT1-transduced cells $(0.16 \pm 0.02 \mathrm{~g}, n=5)$ or shNFAT2-transduced cells $(0.21 \pm 0.01 \mathrm{~g}, n=3)$ cells versus control (LKO) $4 \mathrm{~T} 1$ cells $(0.40 \pm 0.02 \mathrm{~g}, n=5)$. Data are represented as mean \pm S.E.M. (b) Quantification of lung colonization after excision, fixation, paraffin embedding and $\mathrm{H} / \mathrm{E}$ staining of lung sections of mice described in (a). Surface area of metastatic foci and surface of lungs were measured using ImageJ software. Data are represented as mean \pm S.E.M. (c) Representative pictures of lung sections as described in $\mathbf{b}$ after staining for cleaved caspase 3 and Hematoxilin/Eosin. Insets show a higher magnification of the underlined square. Note the brown CC3-positive cells specifically in shCnB, shNFAT1 and shNFAT2 metastatic foci. (d) Quantification of the number of apoptotic cells (CC3+) in metastatic foci of lungs, 15 days after intravenous injection of 4 T1 cells. Data are plotted as fold increase compared with control and are the means of three independent tumors $(n=3$, data are represented as mean \pm S.E.M. $)$

shNFAT1-4T1 cells to degrade the extracellular matrix, an essential process for tumor cells to invade and disseminate, is associated with the decreased NFAT1-dependent expression of ADAMTS1.

\section{Discussion}

Although previously reported data showed a role for NFAT1 in breast cancer cell lines migration in vitro, it remained unclear whether the Cn/NFAT pathway is actually activated in breast cancer and whether this pathway is functionally relevant to breast cancer tumorigenesis. Our results provide the first evidence that the Cn/NFAT pathway is activated in diagnostic cases of breast cancer, with a preferential activation in $~ 50 \%$ in triple-negative subtypes. Targeting the Cn/NFAT pathway could thus be of therapeutic value in this aggressive subtype. Indeed, our results using triple-negative mammary tumor cells demonstrate the essential role of the Cn/NFAT pathway to both the tumorigenic and metastatic potential of these cells in mice. Global gene expression analyses highlighted several major cellular functions that are altered in 4T1 cells in which NFAT1 expression is decreased. In particular, we demonstrated that the gene encoding the protease ADAMTS1 is a direct target of NFAT1, and that its NFAT1-dependent regulation likely participates to the pro-invasive properties of $4 \mathrm{~T} 1$ cells.
Previous reported results showed that $\mathrm{Cn}$ inactivation using pharmacological inhibitors did not result in NFAT inactivation, or impaired in vitro invasion. ${ }^{12}$ Thus, the role of Cn/NFAT pathway in tumor cell invasion remained unclear. Our findings showing that $\mathrm{Cn}$ inactivation through shRNA-mediated silencing of the expression of its $\mathrm{CnB} 1$ regulatory subunit results in NFAT rephosphorylation show that the Cn/NFAT pathway is active in $4 \mathrm{~T} 1$ cells both in vitro and in vivo and that NFAT nuclear translocation relies on $\mathrm{Cn}$ activity. Similar results were found in human breast cancer cell lines (unpublished observation). Moreover, we demonstrate that $\mathrm{Cn}$ is essential in vivo to the tumorigenic and metastatic potential of $4 \mathrm{~T} 1$ cells, thus favoring a pro-oncogenic role of $\mathrm{Cn}$ in disease progression in mammary carcinogenesis. Of note, the ORAl1-3 pore subunit of CRAC, which is an upstream regulator of NFAT activation in other cell types ${ }^{19}$ are involved in breast tumor cell migration and metastasis ${ }^{20}$ and are candidates as upstream activator for Cn. Previous data have linked NFAT1 to in vitro migration and invasion of mammary cancer cell lines. ${ }^{11,12}$ Our loss-of-function studies show that NFAT1 or NFAT2 silencing is sufficient to alter tumorigenesis and metastasis. This may reflect a non-redundant function of these factors in breast carcinogenesis or reflect their mutual dependence in gene expression or protein function. For example, expression of the 

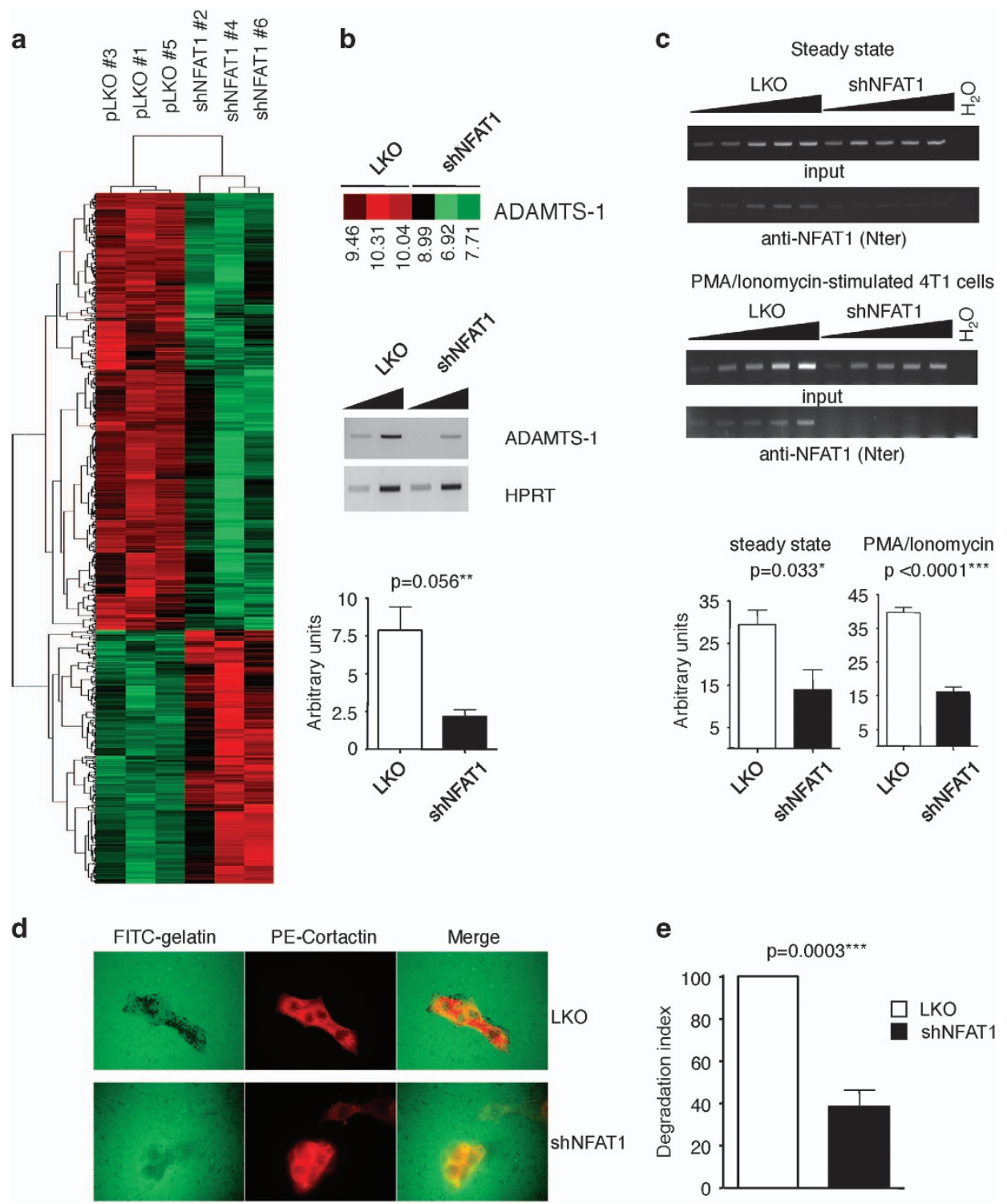

Figure 5 ADAMTS1 expression is downregulated in shNFAT1-4T1 cells. (a) Microarray analysis of three independent cultures of control (pLKO-transduced 4T1 cells) and shNFAT1-transduced 4T1 cells. Hierarchical clustering of the indicated 4T1 cells (top) in their control (pLKO) and NFAT1-silenced versions (shNFAT1) was performed as described in Materials and Methods. The heatmap representation highlights upregulated genes in red and downregulated genes in green. (b) ADAMTS1 is downregulated in shNFAT1-4T1 cells (Java Tree View extract of data shown in a, top panel). Semi-quantitative reverse transcription-PCR analysis of ADAMTS1 expression (middle panel) and quantification in lower panel are shown. HPRT was used to normalize the experiments. Quantification was made of three independent experiments ( $n=3$, data are represented as mean \pm S.E.M.). (c) ChIP/PCR detection of ADAMTS1 promoter in chromatin obtained from 4T1 control (pLKO) and NFAT1-silenced (shNFAT1) 4T1 cells maintained either under steady state (upper panels) or stimulated with PMA/lonomycin for $6 \mathrm{~h}$ to acutely activate NFAT (lower panels). Note the absence of detection of ADAMTS1 promoter coimmunoprecipitated with the anti-NFAT1 antibody in shNFAT1-4T1 cells. The histogram shows the quantification of the co-immunoprecipitated DNA in the different conditions used $(n=5$, data are represented as mean \pm S.E.M.). (d) NFAT1-silenced 4T1 cells cultured for $24 \mathrm{~h}$ on FITC-gelatin show decreased in situ protease activity compared with control 4T1 (pLKO) cells. (e) Quantification of experiments shown in $\mathbf{d}$, as described in Materials and Methods. Data are represented as normalized degradation (degradation index), which was calculated as the area of degraded matrix per cell relative to control pLKO-4T1 cells ( $n=4$, data are represented as mean \pm S.E.M.)

NFAT2/A isoform is under NFAT transcriptional control in T cells. ${ }^{21}$ Such a mechanism does not operate in 4T1 cells, as knockdown of NFAT1 failed to impinge on the expression of any of the NFAT2 isoforms (Figure 2a). Transcriptional regulation by NFAT factors in normal cell physiology most often involves their cooperation with unrelated transcriptional regulators, ${ }^{3}$ or their binding as homodimers to palindromic or close to palindromic response elements. ${ }^{22,23}$ It is thus possible that critical NFAT-dependent genes in mammary tumor cells rely on the binding of NFAT1/NFAT2 heterodimers. Alternatively, NFAT1 and NFAT2 may have completely non-redundant functions in breast cancer and each regulate a set of specific genes, although our results showing that NFAT1 or NFAT2 silencing often results in similar gene deregulation does not favor this last hypothesis.

Epithelial-mesenchymal transition (EMT) is thought to be essential for tumor cells to disseminate from the primary tumor, intravasate and survive into the blood to finally 
extravasate and colonize secondary organs. Activation of Cn catalytic subunit has recently been described to be associated with the acquisition of mesenchymal properties of MCF7 cells on mitochondrial stress, ${ }^{24}$ whereas NFAT could participate to the TGF $\beta$-induced EMT of MDA-MB-231 cells. ${ }^{25}$ We thus investigated whether NFAT1-silenced 4T1 cells presented a more epithelial phenotype compared with controls cells. However, the expression of neither E-cadherin, ESRP1 (epithelial markers) nor that of Snail, Twist, Fibronectin, Vimentin, Mmp9 (mesenchymal markers/inducers) were significantly deregulated in NFAT1-silenced cells (Supplementary Table 3). However, as the highly tumorigenic and metastatic potential of $4 \mathrm{~T} 1$ may not rely on $\mathrm{EMT}^{26}{ }^{26}$ the requirement for Cn/NFAT for metastasis reported here likely relies on mechanisms other than EMT. For instance, apoptosis was clearly found enhanced in tumors and metastatic foci with impaired Cn/NFAT pathway, a phenotype that is associated with the deregulation of a number of genes implicated in apoptosis (Table 1 and Table 2). Further studies will be required to confirm and assess the importance of the

Table 1 Top molecular pathways deregulated in shNFAT1 knocked down cells

\begin{tabular}{lc}
\hline Molecular and cell function & Significance \\
\hline Cellular movement & $3.00 \times 10^{-8}$ \\
Cell death and survival & $1.94 \times 10^{-7}$ \\
Cellular development & $2.88 \times 10^{-7}$ \\
Cellular growth and proliferation & $2.88 \times 10^{-7}$ \\
Cellular function and maintenance & $3.47 \times 10^{-5}$ \\
\hline
\end{tabular}

The 325 genes that showed an at least twofold expression change between control and shNFAT1 samples were submitted to pathway analysis using the Ingenuity Pathways Analysis (IPA). deregulation of these genes in the Cn/NFAT-dependent phenotypes in mammary tumors.

We identified ADAMTS1 as a likely direct NFAT1 target involved in ECM degradation. ADAMTS1 encodes a protease highly upregulated in MDA-MB-231 subclones endowed with high metastatic potential. ${ }^{27}$ It has also been shown essential to mammary tumorigenesis in PyMT model of mammary tumor development ${ }^{14}$ and to metastasis in xenografted MDAMB-231 cells. ${ }^{13}$ Interestingly, the decreased tumorigenesis observed in PyMT/ADAMTS1 $1^{-/}$mice was characterized by increased apoptosis. ${ }^{14}$ It is thus tempting to speculate that decreased ADAMTS1 expression seen in NFAT1-silenced tumors contributes to their apoptotic phenotype. Yet, the relative importance of apoptosis versus invasion/migration in the Cn/NFAT-dependant tumorigenic process remains to be dissected. Our findings provide a new insight into the molecular mechanism underlying the regulation of ADAMTS1 in breast cancer through the Cn/NFAT pathway. As immunotherapy using an anti-ADAMTS1 antibody has been reported to be efficient against $4 \mathrm{~T} 1$-induced tumorigenesis in mice, ${ }^{28}$ our study reinforces the idea of therapeutically targeting ADAMTS1 - and other NFAT targets - to prevent breast cancer metastasis.

Besides NFAT target genes or CRAC inhibitors, the Cn/ NFAT pathway offers other possibilities for therapeutic intervention, for example, by direct targeting of $\mathrm{Cn}$ itself using $\mathrm{Cn}$ inhibitors such as cyclosporine A (CsA) or Tacrolimus (FK506). Recent studies have shown that treatment of MMTV-Neu transgenic mice with FK506 inhibited tumor growth, an effect that was ascribed to impaired NFAT4dependent tumor angiogenesis. ${ }^{29}$ Our results suggest that part of the antitumorigenic effects of FK506 in this model also

Table 2 The top 10 downregulated and upregulated genes with a $P$-value $<0.05$ and ascribed to 'apoptosis' are shown

\begin{tabular}{|c|c|c|}
\hline Gene's name & Log ratio & Relation to breast cancer \\
\hline WISP1* & -2.912 & $\begin{array}{l}\text { Induction of WISP1 correlates with invasive breast cancer oncogenesis and reduced type } 1 \text { cell-mediated cytotoxic } \\
\text { immunity: a retrospective study (Klinke et al., }{ }^{32} \text { ) }\end{array}$ \\
\hline SERPINE1* & -2.295 & Plasma PAI-1 levels in breast cancer-relationship with clinical outcome (Ferroni et al., ${ }^{33}$ ) \\
\hline FST* $^{*}$ & -2.073 & FST found upregulated in highly invasive MDS-MB-435 overexpressing alpha4beta6 integrins $\left(\mathrm{Chen}^{34}\right)$ \\
\hline ADAMTS1* & -2.067 & The ADAMTS1 protease gene is required for mammary tumor growth and metastasis (Ricciardelli et al., ${ }^{14}$ ) \\
\hline NRP1* & -2.033 & $\begin{array}{l}\text { Neuropilin- } 1 \text { is expressed by breast cancer stem-like cells and is linked to NFkB activation and tumor sphere formation } \\
\text { (Glinka et al., }{ }^{35} \text { ) }\end{array}$ \\
\hline NTS* & -2.024 & The neurotensin receptor-1 pathway contributes to human ductal breast cancer progression (Dupouy et al., ${ }^{36}$ ) \\
\hline ROR1* & -1.970 & ROR1 is expressed in human breast cancer and associated with enhanced tumor cell growth (Zhang et al., ${ }^{37}$ ) \\
\hline ADAM12* & -1.940 & $\begin{array}{l}\text { ADAM12 redistributes and activates MMP-14, resulting in gelatin degradation, reduced apoptosis and increased tumor } \\
\text { growth (Albrechtsen et al., }{ }^{38} \text { ) }\end{array}$ \\
\hline DAG1 & -1.696 & \\
\hline ABCG2* & -1.510 & Breast cancer resistance protein \\
\hline IRF8* & 1.616 & Epigenetic inactivation of IRF8 in breast carcinoma (among other carcinoma lines; Lee et al., ${ }^{39}$ ) \\
\hline IFI16 & 1.663 & $\begin{array}{l}\text { Differential regulation of estrogen receptor a expression in breast cancer cells by metastasis-associated protein } 1 \\
\text { (Kang et al.., }{ }^{40} \text { ) }\end{array}$ \\
\hline HIST1H1C & 1.799 & \\
\hline $\begin{array}{l}\text { ITGA1 } \\
\text { NPTX1 }\end{array}$ & $\begin{array}{l}1.875 \\
1.890\end{array}$ & \\
\hline $\mathrm{CXCR}^{*}$ & 2.004 & $\begin{array}{l}\text { C-X-C ligand } 10 \text { and C-X-C receptor } 3 \text { status can predict tamoxifen treatment response in breast cancer patients } \\
\text { (Hilborn et al., }{ }^{41} \text { ) }\end{array}$ \\
\hline $\begin{array}{l}\text { KLF2* } \\
\text { EYA2 } \\
\text { TRIB22 }\end{array}$ & $\begin{array}{l}2.217 \\
2.316 \\
2.414\end{array}$ & Silencing of KLF2 by the histone methyltransferase EZH2 in human cancer (Taniguchi et al., ${ }^{42}$ ) \\
\hline TXNIP* & 2.680 & Role of thioredoxin reductase 1 and thioredoxin interacting protein in prognosis of breast cancer (Cadenas et al., ${ }^{43}$ ) \\
\hline
\end{tabular}

The asterisk indicates that the gene has been associated with breast cancer in the literature. Column 2 indicates the log ratio (In2) measured in the Affymetrix screen Column 3 lists one reference of the link between the indicated gene and breast cancer. NA, not applicable. 
likely resulted from the inhibition of $\mathrm{Cn}$ in the tumor cells themselves. Interestingly, a significant reduction in the incidence of breast cancer was found in a retrospective clinical study of patients treated with CsA after renal and cardiac transplantation. ${ }^{30}$ This difference was ascribed to the impaired supportive function of immune stromal cells to tumor cells but may also reflect direct effects on tumor cells in response to $\mathrm{Cn}$ inhibition by CsA. However, besides induction of secondary cancers, ${ }^{30}$ CsA or FK506 are associated with a number of illcharacterized off-target effects that may limit their usefulness. The identification of upstream activators or downstream effectors of the CnB/NFAT pathway, as reported here, may represent a therapeutical alternative to these limitations.

\section{Materials and Methods}

Cell culture and knockdown. 4T1 were maintained in cultured for $>3$ weeks in DMEM/F12 medium supplemented with $10 \%$ FBS. The two CnB1, NFAT1 and NFAT2-specific shRNA and the control vector (with unrelated shRNA LKO \#71336) were from Open Biosystems/Thermo Scientific (GE Healthcare, Velizy, France) (LKO shCnB11 \#487, GIPZ \#131430; pLKO shNFAT1\#12356, \#12354; pLKO shNFAT2 \#81925, \#81926). Viral production was performed as previously described. ${ }^{10} 4 \mathrm{~T} 1$ cells were selected with puromycin $(1 \mu \mathrm{g} / \mathrm{ml})$ for 5 days.

Migration/invasion assays. Matrigel invasion inserts and migration inserts (8- $\mu \mathrm{m}$ pores) for 24-well tissue culture plates (BD Biosciences, Le Pond-de-Claix, France) were used as recommended by the manufacturer. $10 \%$ FBS was used as chemoattractant using $5 \times 10^{4}$ cells (for migration assays) or $2 \times 10^{5}$ cells (for invasion assays). Migrating cells were fixed with $20 \%$ ethanol and stained with $0.1 \%$ crystal violet (Sigma-Aldrich, St. Louis, MO, USA). For quantification of invasion/ migration, the total area of stained cells was measured using the Threshold command of MetaMorph 6.2.6 (MDS Analytical Technologies, Sunnyvale, CA, USA) and divided by the total surface of the membrane (\% of filled area).

Fluorescent gelatin degradation assays. In total, $3 \times 10^{4} 4 \mathrm{~T} 1$ cells per 12-well plates were incubated for $16 \mathrm{~h}$ on FITC-conjugated cross-linked gelatin (Molecular Probes, Invitrogen, Cergy Pontoise, France) as previously described, ${ }^{31}$ fixed and processed for IF as previously described. ${ }^{10}$ Cells were imaged with the $\times 63$ objective of a Leica DPRXA microscope equipped with a photometrics coolSNAP HQ camera and steered by Metamorph (Molecular Devices Corp., Sunnyvale, CA, USA). The total surface of the coverslip was screened for dark spots specific to matrix degradation that was divided by the total number of cells (cortactin positive) to define a degradation index. The degradation index of control LKO-4T1 cells was set as $100 \%$.

In vivo tumor models. BALB/c mice were injected orthotopically in the 4th mammary gland with $5 \times 10^{5} 4 \mathrm{~T} 1$ cells or its derivatives in $50 \mu \mathrm{l}$ of PBS. Tumor size was measured every 3 days using a caliper and diameter was calculated as $\left(W^{2} \times L\right) / 2$ where $W=$ width and $L=$ length. Mice were killed after 3-6 weeks. For intravenous injection, $5 \times 10^{5} 4 \mathrm{~T} 1$ cells were injected in the lateral tail vein and mice killed 15 days after injection. BalB/C mice were maintained under specific pathogenfree conditions in the animal facility of the Institut Curie. All experimental procedures were performed in accordance with the recommendations of the European Community (86/609/EEC) and the French National Committee (87/848) for the care and use of laboratory animals. All animal experiments were carried out under the supervision of JG, who was authorized by the director of the Veterinary Services of the Préfecture de l'Essonne, Evry, France (agreement number 91-7).

Human samples and clinical and tissue microarray data. Samples of primary breast tumors surgically removed prior to any radiation, hormonal or chemotherapy treatment at Institut Curie from 2005 to 2006 have been analyzed. The clinical and pathological features of patients are summarized (Supplementary Table 1). Our series of diagnostic cases include 321 invasive ductal carcinoma representative of each molecular subtype of breast cancer defined as: luminal $\mathrm{A}(n=101)$ : estrogen receptor (ER) $>10 \%$, progesterone receptor (PR) $>20 \%$, Ki67 $<14 \%$; Luminal B $(n=85)$ : ER $>10 \%$, $\mathrm{PR}<20 \%$, Ki67 $>14 \%$; HER2': ER $<10 \%, \mathrm{PR}<10 \%$, HER2 $2+$ amplified $(n=52) ;$ TNBC (triple negative, $n=83$ ): $\mathrm{ER}<10 \%, \mathrm{PR}<10 \%$, HER2 $0 / 1$ or $2+$ non amplified according to ASCO guideline. TMA consisted of replicate of tumor core selected from whole-tumor tissue section of each tumor sample and a matched tissue core from adjacent non tumoral breast epithelium. Immunohistochemistry was performed using the Leica Bond-III automated immunostainer (Leica Microsystems, Nanterre, France) and the antiNFAT2 antibody (Santa Cruz, Heidelberg, Germany; sc-7294) as previously described. ${ }^{9}$

Analyses were performed in accordance with the French bioethics law 2004-800 and the French National Institute of Cancer (INCa) Ethic Charter and after approval by the Institut Curie board and ethics committee, which waived the need for written informed consent from the participants. Women were informed of the research use of their tissues and did not declare any opposition for such search. Data were analyzed anonymously.

Immunohistochemistry. Seven micrometer sections of paraffin-embedded organs were used for immuno-histological analysis using rabbit anti-NFAT1 (HPA008789, Sigma-Aldrich), rabbit anti-cleaved caspase 3 (mAb \#9664; Cell Signaling Technologies, Leiden, the Netherlands), mouse anti Ki67 (KI67-MM1-CE$S$ Leica) and amplified using the Vectastain Elite $A B C$ kit (Vector) and revealed with DAB Peroxydase Substrate Kit (Vector, Les Ulis, France). Analysis/counting was performed independently by two investigators.

Affymetrix microarrays. Microarray analyses were performed using total RNA from three independent LKO-control and three independent shNFAT-1-4T1 cultures using the Murine Genome 430.2 array (Affymetrix, Santa Clara, CA, USA) according to manufacturer's instructions (http://www.microarrays.ustrasbg.fr). Raw feature data were normalized, and $\log _{2}$ intensity expression summary values for each probe set were calculated using robust multiarray average (package affy V1.4.32). Unsupervised hierarchical clustering analysis was performed using Java Tree view and Cluster programs.

Immunoblotting. Antibodies used in this study are: mouse monoclonal antibodies, anti-NFAT1 4G6-G5 (Santa Cruz, sc-7294), anti-NFAT2 7A6 (Santa Cruz, sc-7294), anti-CnB1 (Sigma-Aldrich, CO581), anti-STAT5 C17 (Santa Cruz).

Semi-quantitative RT-PCR analysis. Total RNA was isolated from $3 \times 10^{6}$ cells using Trizol reagent (Invitrogen). In all, $0.5-1 \mu \mathrm{g}$ of RNA was reverse transcript using the kit ImProm II Reverse Transcription System (Promega, Charbonnieres, France) according to the manufacturer's instructions. PCR were performed using two increasing doses of $C D N A$ as indicated by the increment sign in the figures. GOTaq DNA Polymerase (Promega) and the following protocols were used: $94^{\circ} \mathrm{C}$ for $5 \mathrm{~min}$, followed by 30 cycles of $94^{\circ} \mathrm{C}$ for $1 \mathrm{~min}, 60^{\circ} \mathrm{C}$ for $1 \mathrm{~min}$ and $72{ }^{\circ} \mathrm{C}$ for $1 \mathrm{~min}$. The sequence of the primers used for amplification were as follows: ADAMTS1 forward: 5'-CAGTACCAGACCTTGTGCAGACCTT-3'; ADAMTS1 reverse: 5'-CACACCTCACTGCTTACTGGTTTGA-3'; HPRT forward: 5'-GCTGGTGAAAAGGA CCTC-3', HPRT reverse 5'-CACAGGACTAGACCTGC-3'; ROR1 forward 5'-CCCGA TTTCCCAATTACATG-3'; ROR1 reverse 5'-AGATCGCTGGTTTCATTGGC-3'; FST forward 5'-ACCTGAGAAAGGCCACCT-3'; FST reverse 5'-AGCTTCCTTCATGG CACACT-3'; TXNIP forward 5'-CATGAGGCCTGGAAA-CAAAT-3'; TXNIP reverse 5'-ACTGGTGCCATTAGGTCAGG-3'; KLF2 forward 5'-GCCTGTGGGTTCGCTATA AA-3'; KLF2 reverse 5'-TTTCCCACTTGGGATACAGG-3'.

Chromatin immunoprecipitation. Control and shNFAT1-4T1 cells were stimulated with PMA $(25 \mathrm{ng} / \mathrm{ml})$ and ionomycin $(1 \mathrm{mg} / \mathrm{ml})$ for $4 \mathrm{~h}$, then fixed in $1 \%$ formaldehyde for $20 \mathrm{~min}$ at $37^{\circ} \mathrm{C}$ and processed for chromatin immunoprecipitation as previously described (Lesault, 2002 \#63) using $10 \mu \mathrm{g}$ of anti-NFAT1 antibody (Sigma-Aldrich). The presence of ADAMTS1 promoter fragments in immunoprecipitated chromatin was detected by PCR using $5^{\prime}$-CGCTTTAGCCATGGT GCCCATGG-3' and 5'-CGAAACAGCGCTGGGACCAGC-3' as primers. PCR products were analyzed on agarose gel and quantified using ImageJ software. Unprecipitated DNA was used as input.

\section{Conflict of Interest}

The authors declare no conflict of interest.

Acknowledgements. We thank A Mensah for the analysis of the $\mathrm{HC}$, $\mathrm{H}$ Alcalde for IV injection of the 4T1 cells, M Hirondelle for help with the gelatin assays, $\mathrm{F}$ Cordelières for assistance in cell migration analyses, Vonick Sibut for assistance in transcriptomic analyses, Elodie Belloir and Christophe Alberti for 
assistance with mouse husbandry, Morgane Thion and Sandrine Humbert for discussions. DP was supported by predoctoral fellowships from the Région lle-deFrance and Fondation ARC pour la recherche sur le cancer. This work was supported by funds from the Centre National de la Recherche Scientifique (CNRS), Institut National de la Santé et de la Recherche Médicale (INSERM), Institut Curie and Ligue Nationale Contre le Cancer (Equipe labellisée Ligue).

1. Polyak K. Heterogeneity in breast cancer. J Clin Investig 2011; 121: 3786-3788.

2. Macian F. NFAT proteins: key regulators of T-cell development and function. Nat Rev2005; 5 : 472-484.

3. Muller MR, Rao A. NFAT immunity and cancer: a transcription factor comes of age. Nat Rev 2010; 10: 645-656.

4. Neal JW, Clipstone NA. A constitutively active NFATc1 mutant induces a transformed phenotype in 3T3-L1 fibroblasts. J Biol Chem 2003; 278: 17246-17254.

5. Buchholz M, Schatz A, Wagner M, Michl P, Linhart T, Adler G et al. Overexpression of c-myc in pancreatic cancer caused by ectopic activation of NFATc1 and the $\mathrm{Ca} 2+/$ calcineurin signaling pathway. EMBO J 2006; 25: 3714-3724.

6. Koenig A, Linhart T, Schlengemann K, Reutlinger K, Wegele J, Adler G et al. NFAT-induced histone acetylation relay switch promotes $c$-Myc-dependent growth in pancreatic cancer cells. Gastroenterology 2010; 138: 1189-1199 e1181-1182.

7. Lehen'kyi V, Flourakis M, Skryma R, Prevarskaya N. TRPV6 channel controls prostate cancer cell proliferation via $\mathrm{Ca}(2+) / \mathrm{NFAT}-$ dependent pathways. Oncogene 2007; 26: 7380-7385.

8. Marafioti T, Pozzobon M, Hansmann ML, Ventura R, Pileri SA, Roberton H et al. The NFATc1 transcription factor is widely expressed in white cells and translocates from the cytoplasm to the nucleus in a subset of human lymphomas. Br J Haematol 2005; 128: 333-342.

9. Medyouf H, Alcalde H, Berthier C, Guillemin MC, dos Santos NR, Janin A et al. Targeting calcineurin activation as a therapeutic strategy for T-cell acute lymphoblastic leukemia. Nat Med 2007; 13: 736-741.

10. Gachet S, Genesca E, Passaro D, Irigoyen M, Alcalde H, Clemenson $C$ et al. Leukemiainitiating cell activity requires calcineurin in T-cell acute lymphoblastic leukemia. Leukemia 2013; 27: 2289-2300.

11. Yoeli-Lerner M, Yiu GK, Rabinovitz I, Erhardt P, Jauliac S, Toker A. Akt blocks breast cancer cell motility and invasion through the transcription factor NFAT. Mol Cell 2005; 20: 539-550.

12. Jauliac S, Lopez-Rodriguez C, Shaw LM, Brown LF, Rao A, Toker A. The role of NFAT transcription factors in integrin-mediated carcinoma invasion. Nat Cell Biol 2002; 4: 540-544

13. Lu X, Wang Q, Hu G, Van Poznak C, Fleisher M, Reiss M et al. ADAMTS1 and MMP1 proteolytically engage EGF-like ligands in an osteolytic signaling cascade for bone metastasis. Genes Dev 2009; 23: 1882-1894.

14. Ricciardelli C, Frewin KM, Tan Ide A, Williams ED, Opeskin K, Pritchard MA et al. The ADAMTS1 protease gene is required for mammary tumor growth and metastasis Am J Pathol 2011; 179: 3075-3085.

15. Rashid OM, Nagahashi M, Ramachandran S, Dumur Cl, Schaum JC, Yamada A et al. Is tail vein injection a relevant breast cancer lung metastasis model? J Thorac Dis 2013; 5 : 385-392.

16. Doyle KM, Russell DL, Sriraman V, Richards JS. Coordinate transcription of the ADAMTS-1 gene by luteinizing hormone and progesterone receptor. Mol Endocrinol 2004; 18 2463-2478.

17. Liu YJ, Xu Y, Yu Q. Full-length ADAMTS-1 and the ADAMTS-1 fragments display pro- and antimetastatic activity, respectively. Oncogene 2006; 25: 2452-2467.

18. Lind T, Birch MA, McKie N. Purification of an insect derived recombinant human ADAMTS-1 reveals novel gelatin (type I collagen) degrading activities. Mol cell Biochem 2006; 281 95-102.

19. Feske S, Gwack Y, Prakriya M, Srikanth S, Puppel SH, Tanasa B et al. A mutation in Orai1 causes immune deficiency by abrogating CRAC channel function. Nature 2006; 441 : 179-185.

20. Yang S, Zhang JJ, Huang XY. Orai1 and STIM1 are critical for breast tumor cell migration and metastasis. Cancer Cell 2009; 15: 124-134.

21. Chuvpilo S, Jankevics E, Tyrsin D, Akimzhanov A, Moroz D, Jha MK et al. Autoregulation of NFATc1/A expression facilitates effector $T$ cells to escape from rapid apoptosis. Immunity 2002; 16: 881-895.

22. Jin L, Sliz P, Chen L, Macian F, Rao A, Hogan PG et al. An asymmetric NFAT1 dimer on a pseudo-palindromic kappa B-like DNA site. Nat Struct Biol 2003; 10: 807-811.

23. Soto-Nieves N, Puga I, Abe BT, Bandyopadhyay S, Baine I, Rao A et al. Transcriptional complexes formed by NFAT dimers regulate the induction of T cell tolerance. J Exp Med 2009; 206: 867-876.

24. Guha M, Srinivasan S, Ruthel G, Kashina AK, Carstens RP, Mendoza A et al. Mitochondrial retrograde signaling induces epithelial-mesenchymal transition and generates breast cancer stem cells. Oncogene 2013; 33: 5238-5250.
25. Sengupta S, Jana S, Biswas S, Mandal PK, Bhattacharyya A. Cooperative involvement of NFAT and SnoN mediates transforming growth factor-beta (TGF-beta) induced EMT in metastatic breast cancer (MDA-MB 231) cells. Clin Exp Metast 2013; 30: 1019-1031.

26. Lou Y, Preobrazhenska O, auf dem Keller U, Sutcliffe M, Barclay L, McDonald PC et al. Epithelial-mesenchymal transition (EMT) is not sufficient for spontaneous murine breast cancer metastasis. Dev Dyn 2008; 237: 2755-2768.

27. Kang Y, Siegel PM, Shu W, Drobnjak M, Kakonen SM, Cordon-Cardo C et al. A multigenic program mediating breast cancer metastasis to bone. Cancer Cell 2003; 3: 537-549.

28. Hirano T, Hirose K, Sakurai K, Makishima M, Sasaki K, Amano S. Inhibition of tumor growth by antibody to ADAMTS1 in mouse xenografts of breast cancer. Anticancer Res 2011; 31 : 3839-3842.

29. Siamakpour-Reihani S, Caster J, Bandhu Nepal D, Courtwright A, Hilliard E, Usary J et al. The role of calcineurin/NFAT in SFRP2 induced angiogenesis-a rationale for breast cancer treatment with the calcineurin inhibitor tacrolimus. PloS One 2011; 6: e20412.

30. Wimmer CD, Angele MK, Schwarz B, Pratschke S, Rentsch M, Khandoga A et al. Impact of cyclosporine versus tacrolimus on the incidence of de novo malignancy following liver transplantation: a single center experience with 609 patients. Transpl Int 2013; 26: 999-1006.

31. Coopman PJ, Do MT, Thompson EW, Mueller SC. Phagocytosis of cross-linked gelatin matrix by human breast carcinoma cells correlates with their invasive capacity. Clin Cancer Res 1998; 4: 507-515.

32. Klinke DJ 2nd. Induction of Wnt-inducible signaling protein-1 correlates with invasive breast cancer oncogenesis and reduced type 1 cell-mediated cytotoxic immunity: a retrospective study. PLoS Comput Biol 2014; 10: e1003409.

33. Ferroni P, Roselli M, Portarena I, Formica V, Riondino S, LA Farina F et al. Plasma plasminogen activator inhibitor-1 (PAl-1) levels in breast cancer - relationship with clinical outcome. Anticancer Res 2014; 34: 1153-1161.

34. Chen M, Sinha M, Luxon BA, Bresnick AR, O'Connor KL. Integrin alpha6beta4 controls the expression of genes associated with cell motility, invasion, and metastasis, including S100A4/metastasin. J Biol Chem 2009; 284: 1484-1494.

35. Glinka Y, Mohammed N, Subramaniam V, Jothy S, Prud'homme GJ. Neuropilin-1 is expressed by breast cancer stem-like cells and is linked to NF-kappaB activation and tumor sphere formation. Biochem Biophys Res Commun 2012; 425: 775-780.

36. Dupouy S, Viardot-Foucault V, Alifano M, Souaze F, Plu-Bureau G, Chaouat M et al. The neurotensin receptor-1 pathway contributes to human ductal breast cancer progression. PLoS One 2009; 4: e4223.

37. Zhang S, Chen L, Cui B, Chuang HY, Yu J, Wang-Rodriguez $\mathrm{J}$ et al. ROR1 is expressed in human breast cancer and associated with enhanced tumor-cell growth. PloS One 2012; 7: e31127.

38. Albrechtsen R, Kveiborg M, Stautz D, Vikesa J, Noer JB, Kotzsh A et al. ADAM12 redistributes and activates MMP-14, resulting in gelatin degradation, reduced apoptosis and increased tumor growth. J Cell Sci 2013; 126: 4707-4720.

39. Lee KY, Geng H, Ng KM, Yu J, van Hasselt A, Cao Y et al. Epigenetic disruption of interferongamma response through silencing the tumor suppressor interferon regulatory factor 8 in nasopharyngeal, esophageal and multiple other carcinomas. Oncogene 2008; 27: 5267-5276.

40. Kang HJ, Lee MH, Kang HL, Kim SH, Ahn JR, Na H et al. Differential regulation of estrogen receptor alpha expression in breast cancer cells by metastasis-associated protein 1. Cancer Res 2014; 74: 1484-1494.

41. Hilborn E, Sivik T, Fornander T, Stal O, Nordenskjold B, Jansson A. C-X-C ligand 10 and $\mathrm{C}-\mathrm{X}-\mathrm{C}$ receptor 3 status can predict tamoxifen treatment response in breast cancer patients. Breast Cancer Res Treat 2014; 145: 73-82.

42. Taniguchi H, Jacinto FV, Villanueva A, Fernandez AF, Yamamoto H, Carmona FJ et al. Silencing of Kruppel-like factor 2 by the histone methyltransferase EZH2 in human cancer. Oncogene 2012; 31: 1988-1994.

43. Cadenas C, Franckenstein D, Schmidt M, Gehrmann M, Hermes M, Geppert B et al. Role of thioredoxin reductase 1 and thioredoxin interacting protein in prognosis of breast cancer. Breast Cancer Res 2013; 12: R44.

(i) Cell Death and Disease is an open-access journal published by Nature Publishing Group. This work is licensed under a Creative Commons Attribution 4.0 International Licence. The images or other third party material in this article are included in the article's Creative Commons licence, unless indicated otherwise in the credit line; if the material is not included under the Creative Commons licence, users will need to obtain permission from the licence holder to reproduce the material. To view a copy of this licence, visit http://creativecommons.org/licenses/by/4.0 